Universidad Nacional Experimental Francisco de Miranda (UNEFM). Santa Ana de Coro. Venezuela

Blanca Nieves Cárdenas-Contreras; Darwin Gabriel García-Herrera; María Isabel Álvarez-Lozano; Juan Carlos Erazo-Álvarez

DOI $10.35381 / \mathrm{cm} . v 6 \mathrm{i} 3.390$

\title{
Aprendizaje Basado en Proyectos una Estrategia Metodológica para la Enseñanza de Ciencias Sociales en Bachillerato
}

\section{Project-based learning a methodological strategy for the teaching of social} sciences in high school

\author{
Blanca Nieves Cárdenas-Contreras \\ blanca.cardenas@psg.ucacue.edu.ec \\ Universidad Católica de Cuenca, Azogues \\ Ecuador \\ https://orcid.org/0000-0001-5941-7263 \\ Darwin Gabriel García-Herrera \\ dggarciah@ucacue.edu.ec \\ Universidad Católica de Cuenca, Azogues \\ Ecuador \\ https://orcid.org/0000-0001-6813-8100 \\ María Isabel Álvarez-Lozano \\ mialvarezl@ucacue.edu.ec \\ Universidad Católica de Cuenca, Cuenca \\ Ecuador \\ https://orcid.org/0000-0001-8029-1933 \\ Juan Carlos Erazo-Álvarez \\ jcerazo@ucacue.edu.ec \\ Universidad Católica de Cuenca, Cuenca \\ Ecuador \\ https://orcid.org/0000-0001-6480-2270
}

Recibido: 20 de agosto de 2020

Aprobado: 15 de noviembre de 2020 
Blanca Nieves Cárdenas-Contreras; Darwin Gabriel García-Herrera; María Isabel Álvarez-Lozano; Juan Carlos Erazo-Álvarez

\title{
RESUMEN
}

El objetivo de la investigación es determinar cómo el Aprendizaje Basado en Proyectos influye en la enseñanza de Ciencias Sociales en bachillerato, recurriendo metodológicamente al enfoque mixto, se analizó, que la estrategia de mayor efectividad en la enseñanza de esta disciplina en bachillerato, se encaminó al ABP, vinculado a recursos didácticos digitales como: Powtoon, Storytelling, Genially y otros. Por medio de su aplicación se motivó a los estudiantes por el hábito de aprender esta asignatura, pero ya no de manera tradicional sino más bien muy creativa y dinámica, donde el estudiante es el constructor de su propio aprendizaje. Situación que se encuentra vigente por motivos de la pandemia del COVID-19 demostrando cual importante es el acompañamiento y afecto de los padres, familiares, docentes y autoridades educativas, con la finalidad de consolidar el gusto por aprender Ciencias Sociales a todo el estudiantado de este nivel.

Descriptores: Proyecto de educación; proyecto del alumno; ciencias sociales. (Palabras tomadas del Tesauro UNESCO).

\begin{abstract}
The objective of the research is to determine how Project-Based Learning influences the teaching of Social Sciences in high school, methodologically resorting to the mixed approach, it was analyzed, that the most effective strategy in teaching this discipline in high school, was aimed at ABP, linked to digital teaching resources such as: PowToon, Storytelling, Genially and others. Through its application, students were motivated by the habit of learning this subject, but no longer in a traditional way but rather very creative and dynamic, where the student is the builder of their own learning. Situation that is in force due to the COVID-19 pandemic, demonstrating how important the accompaniment and affection of parents, relatives, teachers and educational authorities is, in order to consolidate the taste for learning Social Sciences to all the students of this level.
\end{abstract}

Descriptors: Educational projects; student projects; social sciences. (Words taken from the UNESCO Thesaurus). 
Universidad Nacional Experimental Francisco de Miranda (UNEFM). Santa Ana de Coro. Venezuela

Blanca Nieves Cárdenas-Contreras; Darwin Gabriel García-Herrera; María Isabel Álvarez-Lozano; Juan Carlos Erazo-Álvarez

\section{INTRODUCCIÓN}

La sociedad es cambiante y por ende la educación, por ello la necesidad de incorporar nuevas estrategias metodológicas que promuevan el desarrollo de habilidades en nuestros estudiantes, permitiendo enfrentar las exigencias de la globalización que hoy vivimos. En este sentido el presente trabajo de investigación se enfoca de manera específica en el Aprendizaje Basado en Proyectos (ABP) como estrategia metodológica en la enseñanza de Ciencias Sociales en el bachillerato, mismo que según (Valdivia \& Cobo, 2017), señala que es una metodología que se realiza de manera colaborativa, en donde los estudiantes se enfrentan a situaciones problemáticas y buscan plantear alternativas de solución. Esta metodología promueve un aprendizaje activo, autónomo y auto dirigido, en donde los estudiantes construyen su propio aprendizaje.

En tal virtud, la razón que incentivó a realizar el presente trabajo, se basa en la observación directa, que se vive a diario en las instituciones educativas, que, actualmente aún siguen utilizando técnicas y métodos tradicionales en el proceso de interaprendizaje; dando como consecuencia en los alumnos un aprendizaje de tipo mecánico, no razonan, ni reflexionan sobre determinados aspectos históricos; de ahí su rendimiento bajo como uno de los factores desencadenantes de esta problemática. Para solucionar en parte esta situación, se propone implementar nuevas estrategias, como el Aprendizaje Basado en Proyectos, que permite al estudiante pensar, razonar y buscar posibles soluciones a los problemas de su diario vivir, preparándolo de esta manera a que puedan desenvolverse en la sociedad sin ningún inconveniente.

Ante esta situación según informes analizados por (León, Martinez, \& Santos, 2018), de la investigación sobre Aprendizaje Basado en Proyectos en el marco europeo se comprueba que las últimas tendencias pedagógicas se encaminan por dejar atrás las clases del modelo tradicional de enseñanza, para potenciar un aprendizaje constructivista, activo, social y conectado con la vida del estudiante. Es decir, aplicar esta 
Universidad Nacional Experimental Francisco de Miranda (UNEFM). Santa Ana de Coro. Venezuela

Blanca Nieves Cárdenas-Contreras; Darwin Gabriel García-Herrera; María Isabel Álvarez-Lozano; Juan Carlos Erazo-Álvarez

metodología facilitará a que, los docentes cambien los conocimientos teóricos en prácticos, mejorando de esta manera la educación, considerando que países desarrollados han aplicado esta metodología dejando de lado la pedagogía tradicional. Según estudios realizados a nivel de Latinoamérica, especialmente en Colombia, se evidencia que las pruebas o cuestionarios sirven para verificar el grado de asimilación de conocimientos que tiene el estudiante puesto que no, existe una justificación de tipo científico sobre la eficacia de la utilización del ABP como estrategia metodológica de enseñanza dentro de la educación, por ello el Ministerio de Educación se preocupa en superar esta falencia, motivando a la implementación por parte de los docente de nuevas estrategias metodológicas activas, una de ellas la aplicación de un proyecto, basado en la metodología $\mathrm{ABP}$, donde se puede comprobar la existencia de una ruptura tradicional de enseñanza, demandando cambios en toda la comunidad educativa, como lo indica (Ciro, 2012)

En cambio, en el Ecuador mediante el análisis bibliográfico se ha determinado, que el bajo rendimiento de los estudiantes en el área de Ciencias Sociales se debe a la falta de implementación de estrategias metodológicas activas, por parte de los docentes, mismos que carecen de una capacitación constante, por lo tanto, siguen manteniendo la enseñanza tradicional, de ahí surge el desinterés del estudiante por temas históricos, si aprende, lo hace más por cumplir.

Por ello el (Ministerio de Educación del Ecuador, 2019) preocupado de esta situación, ha diseñado para los estudiantes de los primeros y segundos años de bachillerato, como requisito para graduarse del Programa de Participación Estudiantil (PPE), en donde se trabaja en proyectos que dan respuesta a problemáticas sociales. En tal virtud muchos países de Latinoamérica deben tomar las iniciativas de otros sistemas educativos como Europa y Colombia que aplicaron en sus prácticas educativas esta metodología, obteniendo resultados muy positivos, mejorando la calidad de educación. 
Universidad Nacional Experimental Francisco de Miranda (UNEFM). Santa Ana de Coro. Venezuela

Blanca Nieves Cárdenas-Contreras; Darwin Gabriel García-Herrera; María Isabel Álvarez-Lozano; Juan Carlos Erazo-Álvarez

Además, con la implementación de esta estrategia metodológica en época del Covid-19, de manera virtual se pretende motivar a los estudiantes a despertar su interés por las diferentes actividades que se realiza dentro de las asignaturas de Ciencias Sociales, por lo tanto, el docente se convierte en el guía y asesor para los estudiantes a fin de mejorar sus aprendizajes. Por ello la necesidad de trabajar, con proyectos innovadores como el ABP.

En función de lo expuesto el objetivo de la investigación es determinar como el Aprendizaje Basado en Proyectos influye en la enseñanza de ciencias sociales en bachillerato, en los estudiantes de la Unidad Educativa "Luis Rogerio González".

\section{Referencial teórico}

El Aprendizaje Basado en Proyectos (ABP) dentro de la disciplina de Ciencias Sociales se ha convertido actualmente, en la parte esencial del proceso enseñanza aprendizaje, ya que es una estrategia metodológica que permite la existencia de una interacción dinámica dentro de los salones de clases y a su vez da la oportunidad a los estudiantes a plantearse situaciones problemáticas de la realidad, partiendo de su trabajo y guía docente encontrara posibles soluciones a los problemas planteados en sus proyectos, además contribuye a que los profesores a través de las capacitaciones se innoven y puedan mejorar la calidad de la educación.

Por ello, luego de haber indagado numerosos estudios sobre la aplicación de esta estrategia metodológica del siglo XXI, como lo es el Aprendizaje Basado en Proyectos, en los países desarrollados y en vías de desarrollo se puede deducir que en Estados Unidos y Europa, la aplicación dentro de la educación ha dado muy buenos resultados, convirtiéndose en países industrializados, puesto que su prioridad en el campo educativo es preparar a los estudiantes a ser emprendedores, de ahí que los países de América tratan de seguir ese ejemplo, como es el caso de Cuba, Colombia y México, etc. 
Universidad Nacional Experimental Francisco de Miranda (UNEFM). Santa Ana de Coro. Venezuela

Blanca Nieves Cárdenas-Contreras; Darwin Gabriel García-Herrera; María Isabel Álvarez-Lozano; Juan Carlos Erazo-Álvarez

Que implementaron la metodología obteniendo muy buenos resultados, razón por la cual el Ecuador a través del Ministerio de Educación en sus programas curriculares fortalece el uso adecuado de esta metodología, con la finalidad de mejor la calidad educativa y formar estudiantes activos, críticos, reflexivos y sobre todo listos para emprender en cada una de las habilidades adquiridas durante sus estudios académicos.

Es necesario aclarar que el uso del ABP y otras metodologías deben estar acompañadas de las herramientas digitales que ofrece el mundo tecnológico, de ahí la prioridad de que docente, deben implementar las TIC en las distintas asignaturas pertenecientes a Ciencias Sociales, con el fin de obtener información muy relevante, que permitan mejorar la calidad educativa en la institución de estudio como es la Unidad Educativa Luis Rogerio González de la ciudad de Azogues. (Sierra, Bueno, \& Monroy, 2016).

\section{Aprendizaje Basado en Proyectos}

Es una estrategia metodológica de enseñanza aprendizaje del siglo XXI, en la cual los estudiantes son los protagonistas de la construcción de su propio aprendizaje, realizando proyectos dentro o fuera del salón de clases, permitiendo aplicar los saberes adquiridos acerca de un tema específico, practicando de esa manera el sistema conceptual para resolver los problemas de la vida real como lo indica (Medina \& Tapia, 2017), es decir, esta metodología permite al estudiante realizar un proyecto, desarrolle habilidades y destrezas como, por ejemplo, piense, reflexiona y busque las mejores alternativas de solución al problema planteado, incluso que presente propuestas que van encaminadas a superar dicho inconveniente.

Según (García-Varcálcel \& Basilotta, 2017) esta estrategia metodológica tiene una larga trayectoria, aparece a principios del siglo XX, con una teoría constructivista en donde Dewey destacó la importancia de la experiencia en el aprendizaje y apostaba por proyectos multidisciplinares, donde permitan al estudiante trabajar diferentes conceptos y áreas de conocimiento, dando importancia al aprendizaje social, por lo que sus 
Universidad Nacional Experimental Francisco de Miranda (UNEFM). Santa Ana de Coro. Venezuela

Blanca Nieves Cárdenas-Contreras; Darwin Gabriel García-Herrera; María Isabel Álvarez-Lozano; Juan Carlos Erazo-Álvarez

proyectos tenían un carácter colaborativo, mientras que Kilpatrick también pedagogo defendió esta metodología porque ayuda en el estudiante a mejorar su potencial. Incluso el objetivo principal de la utilización del ABP es la obtención del producto final, es decir con su aplicación.

Ante esta situación (Romero, Forero, \& Rodríguez, 2018) indican que la característica principal del aprendizaje Basado en Proyectos es que es el estudiante realice un trabajo de investigación, mismo que está estructurado por un inicio, un desarrollo y un final, su contenido debe ser significativo, puesto que les da la posibilidad que resuelvan problemas de la vida real. Además, un proyecto debe ser diseñado con un marco teórico en donde se refleja el planteamiento del problema, la justificación, antecedentes y objetivos que va a alcanzar el estudiante al final del trabajo.

Cabe recalcar que esta metodología, ya era utilizada, pero con técnicas tradicionales, e en donde el estudiante realizaba un trabajo práctico en el área de Ciencias Sociales al final del año.

La planificación de un proyecto requiere de bastante tiempo y dedicación, puesto que primero los estudiantes tienen que elegir el tema, y al mismo tiempo estar involucrados en la planificación y organización del mismo, en este caso el docente pasa a ser quien organice los grupos de trabajo y les guía en la elaboración, hasta la presentación final. En donde la evaluación comprenderá la presentación del producto final, que puede ser llevado a la práctica dentro de la institución educativa, de diversas formas donde le permita al estudiante resumir el proceso de su trabajo, mismo que necesita de una planificación antes de presentar a los espectadores, existiendo diferentes formas de evaluar a más la del maestro, como puede ser entre pares y la autoevaluación el docente calificará de acuerdo a un instrumento elaborado con su debida anticipación. (Habók \& Nagy, 2016)

(Cunill, 2017), refiere que en el Aprendizaje Basado en Proyectos el conocimiento no es transmitido por el docente, sino que es el resultado de un trabajo entre estudiantes y 
Universidad Nacional Experimental Francisco de Miranda (UNEFM). Santa Ana de Coro. Venezuela

Blanca Nieves Cárdenas-Contreras; Darwin Gabriel García-Herrera; María Isabel Álvarez-Lozano; Juan Carlos Erazo-Álvarez

profesores, en el que, se formulan preguntas, se realiza una búsqueda de la información y se obtienen conclusiones, participando activamente de procesos cognitivos, mediante la elaboración del proyecto, de igual manera la función del docente es crear situaciones de aprendizaje en donde permite a los estudiantes desarrollar el trabajo, valorar y gestionar el avance del proyecto y a la vez evalúa el resultado, incluso aprecia su autoestima, ayuda a desarrollar un pensamiento crítico sobre el tema y a la vez enseña a trabajar en equipo ,manteniendo una comunicación activa entre todos.

Luego de una investigación realizada por (Molina, 2019) en la formación de los docentes en las aulas universitarias, pudo comprobar que la mayoría de los estudiantes desconocen las nuevas metodologías activas del siglo XXI, por ello propone que desde el inicio se debe ensenar estrategias metodológicas especialmente el Aprendizaje Basado en Proyectos, para encaminar en la búsqueda de habilidades, destrezas y conocimientos garantizando sus aplicaciones prácticas, por ser una asignatura que está relacionada con el espacio histórico y geográfico.

De ahí que los profesores de educación secundaria especialmente de bachillerato consideran que los investigadores educativos desconocen por completo la realidad de las aulas, ya que los estudios que realizan poco pueden aportar en mejorar la práctica educativa. (Murillo \& Perines, 2017)Por ello (Sánchez, 2018) indica que según evidencias científicas los estudiantes que reciben clases magistrales tienen 1,5 veces más probabilidades de fracasar que los estudiantes con materias que promueven el aprendizaje activo, es decir los que se aprenden de memoria o de manera mecánica pronto se olvidan de todos los conocimientos adquiridos, mientras tanto cuando un estudiante recibe su clase activamente y comprende, es más, a veces ellos son los protagonistas, algo que no se van a olvidar, teniendo un aprendizaje a largo plazo, puesto que gracias al avance tecnológico ha existido un cambio de roles entre el estudiante y el docente el primero se convierte en autónomo, en donde ya no va a necesitar de la transmisión de conocimientos, ya que la tecnología le da la oportunidad de hacerlo 
Universidad Nacional Experimental Francisco de Miranda (UNEFM). Santa Ana de Coro. Venezuela

Blanca Nieves Cárdenas-Contreras; Darwin Gabriel García-Herrera; María Isabel Álvarez-Lozano; Juan Carlos Erazo-Álvarez

pasando el docente a ser el guía y facilitador de herramientas pedagógicas que le permitan construir su propio aprendizaje.

\section{Metodologías activas}

Cada época tiene sus trasformaciones, por tal razón es importante que la educación siga innovándose, dejando atrás aquellas metodologías en que el estudiante solo es el receptor, de ahí la importancia de aplicar en la enseñanza aprendizaje nuevas estrategias acordes a la realidad como lo indica (Gómez, Ortuño, \& Millares, 2018), que la enseñanza debe ser práctica, basadas en metodologías innovadoras, dejando atrás aquel sistema de enseñanza basado en el memorismo y propiciar una enseñanza, donde el estudiante de bachillerato con los conceptos básicos desarrollen un pensamiento crítico y reflexivo de los acontecimientos históricos.

Con estas consideraciones, no se trata de enseñar esta asignatura, para formar científicos en el futuro, sino de implementar las mejores herramientas pedagógicas y tecnológicas en la educación, para contribuir a la formación de personas con actitudes y aptitudes que necesitarán a lo largo de su vida estudiantil y profesional, en donde aprendan a utilizar formas de pensamiento histórico y geográfico, de este modo haciendo comprensivo su mundo, por esta razón es tan importante la introducción de metodologías, relacionadas con el entorno social, para aplicar los conocimientos a otras situaciones del pasado o del presente para comprenderlas acertadamente. (Arteaga, Arteaga, \& Martínez, 2016)

En cambio una innovación dentro de la educación demanda de un cuidadoso diseño, ya que los involucrados son los docentes y los estudiantes, por ello, la necesidad de realizar estudios muy profundos antes de aplicar nuevos métodos y técnicas de enseñanza (Ruiz \& Gómez, 2017); ya que todo proyecto debe ser estudiado minuciosamente, y trabajado con mucha cautela, por que se experimenta con seres humanos, de ahí la necesidad, que los docentes reciban capacitaciones constantes a cerca del uso y aplicación de metodologías activas dentro del proceso enseñanza aprendizaje, ya que un maestro 
Universidad Nacional Experimental Francisco de Miranda (UNEFM). Santa Ana de Coro. Venezuela

Blanca Nieves Cárdenas-Contreras; Darwin Gabriel García-Herrera; María Isabel Álvarez-Lozano; Juan Carlos Erazo-Álvarez

innovador debe tener una mente permeable; es decir, estar dispuesto al cambio, y no quedarse con lo aprendido y lo que el Ministerio de Educación le impone, debe ser curioso, indagador positivo e innovador, ya que nuestra educación siempre estará sujeta a cambios y transformaciones (Orozco, 2016)

Por esta razón es muy necesario el uso de herramientas digitales junto con las metodologías activas, en el proceso de enseñanza de ciencias sociales aunque, esta utilización no se realice de manera eficaz; ya que existe todavía reservas sobre su uso, que están muy vinculadas con la formación docente, sin embargo, son escasas las investigaciones sobre la formación del profesorado de Ciencias Sociales, es decir, el uso de herramientas tecnológicas y metodologías depende de su formación y sus habilidades para aplicarlas dentro del salón de clases. (Millares, Gómez, Arias, \& Fontal, 2019) Estas estrategias metodológicas, en la enseñanza de esta disciplina, deben tener como propósito que sea el estudiante el que descubra y asimile el medio en donde vive, atendiendo su complejidad, incluso el profesor debe dejar atrás aquella enseñanza tradicional, y más bien motivar a resolver situaciones que él plantee de su realidad en relación con el tema de estudio (Gómez \& Rodríguez, 2014)

El Aprendizaje Basado en Proyectos tiene como objetivo motivar al estudiante a apropiarse del objeto de estudio y a su vez replantearse descubrimientos, contribuyendo de esa manera a la solución y promoviendo al desarrollo de su autonomía, en donde puede utilizar sus vivencias y experiencias de aprendizaje, promoviendo la libertad del estudiante (Neiva, Bandeira, Alencar, \& Magalhães, 2020)

\section{Diferencias entre educación tradicional y moderna}

Actualmente se observa que la escuela con metodologías antiguas, es incapaz de generar un pensamiento crítico, reflexivo en el estudiante, ya que solo se centra en llenarle de contenidos, pero de manera mecánica, en donde solo él es el receptor pasivo, y no participa activamente, en el proceso enseñanza aprendizaje, ya que se basan solo 
Universidad Nacional Experimental Francisco de Miranda (UNEFM). Santa Ana de Coro. Venezuela

Blanca Nieves Cárdenas-Contreras; Darwin Gabriel García-Herrera; María Isabel Álvarez-Lozano; Juan Carlos Erazo-Álvarez

en la copia y la memorización, no permitiendo de esa manera la participación del alumno en la toma de decisiones, mientas no se cambie con esta ideología en la actualidad, seguiremos formando estudiantes sumisos al sistema, sin dar la oportunidad, que ellos sean los protagonistas de su propio aprendizaje, por ello el bajo rendimiento en las pruebas ser bachiller, mismas que están elaboradas acorde a las metodologías activas del siglo XXI, (Martinez, 2018)

Por ende (Romero, Forero, \& Rodríguez, 2018) Manifiestan que a lo largo del tiempo se ha desarrollado nuevas estrategias metodológicas para la formación de los estudiantes y la apropiación del conocimiento, en donde ellos son, participativos, dinámicos, creativos y protagonistas en relación a su entorno, de ahí que el constructivismo pretende a través de sus experiencias lograr el afianzamiento de ideas cognitivas, a través del Aprendizaje Basado en Proyectos, que busca calidad en la educación, donde el estudiante no solo aprenda contenidos académicos, sino que desarrolla competencias que lleven a enfrentar los retos, dentro de la sociedad.

En un sin número de establecimientos educativos todavía se sigue utilizando metodologías tradicionales, en donde el centro del aprendizaje es el docente y el estudiante el receptor, ya que siguen el plan de estudios al pie de la letra, sin aplicar las tecnologías y metodologías activas acorde a la época.

Sin duda la enseñanza en la actualidad, involucra a los estudiantes, por medio del trabajo colaborativo y cooperativo donde trabajan todos en bienestar del grupo para alcanzar así los objetivos planteados en el proyecto, incluso en la manera de sentarse, en la tradicional el docente indica que tienen que estar uno detrás del otro, en cambio en la moderna se agrupan en un número determinado para que ejecuten las tares. (Ardeleanu, 2019)

\section{METODOLOGÍA}

El siguiente estudio investigativo obedece a un diseño no experimental, de tipo descriptiva transversal, la muestra poblacional estuvo conformada por 205 estudiantes 
Universidad Nacional Experimental Francisco de Miranda (UNEFM). Santa Ana de Coro. Venezuela

Blanca Nieves Cárdenas-Contreras; Darwin Gabriel García-Herrera; María Isabel Álvarez-Lozano; Juan Carlos Erazo-Álvarez

del tercer año de bachillerato y 15 docentes del Área de Ciencias Sociales de la Unidad Educativa Luis Rogerio González del Cantón Azogues - Ecuador.

Se aplicó una encuesta y un cuestionario de 12 preguntas en la escala de Likert, relacionadas con las variables de la investigación planteada, construida a través de la plataforma de Microsoft Forms, siendo difundida al grupo de WhatsApp, tanto para los docentes como para los estudiantes, previo aviso a las autoridades. Se validó el instrumento en un 0,748 del Alfa de Cronbach, tanto para los estudiantes como para los docentes, lo que permitió que el instrumento fuera validado y los datos fiables para la investigación. En lo que hace referencia al desarrollo estadístico de las variables planteadas se utilizó el programa SPSS, facilitando el proceso de análisis de valores y la obtención de datos confiables, siendo presentados descriptivamente.

\section{RESULTADOS}

Luego de ser analizados los datos de las encuestas, se presentan los resultados:

\section{Tabla 1.}

En las clases de Ciencias Sociales, los docentes aplican metodologías activas al momento de enseñar.

\begin{tabular}{cccccc}
\hline & Frecuencia & Porcentaje & Porcentaje válido & $\begin{array}{c}\text { Porcentaje } \\
\text { acumulado }\end{array}$ \\
\hline \multirow{4}{*}{ Válidos } & Muy de acuerdo & 44 & 21,5 & 21,5 & 21,5 \\
& De acuerdo & 127 & 62,0 & 62,0 & 83,4 \\
& Indeciso & 30 & 14,6 & 14,6 & 98,0 \\
& En desacuerdo & 4 & 2,0 & 2,0 & 100,0 \\
& Total & 205 & 100,0 & 100,0 & \\
\hline
\end{tabular}

Fuente: Elaboración propia.

La tabla 1, muestra que el $83,4 \%$ están muy de acuerdo y de acuerdo en que sus docentes aplican metodologías activas al momento de impartir sus clases; mientras que el $16,6 \%$ manifiestan estar indecisos y en desacuerdo en este aspecto, porcentaje 
Universidad Nacional Experimental Francisco de Miranda (UNEFM). Santa Ana de Coro. Venezuela

Blanca Nieves Cárdenas-Contreras; Darwin Gabriel García-Herrera; María Isabel Álvarez-Lozano; Juan Carlos Erazo-Álvarez

preocupante por cuanto los estudiantes deberían conocer los métodos lo que sus docentes aplican.

\section{Tabla 2.}

El aprendizaje Basado en proyectos es una estrategia metodológica que fomenta la elaboración de proyectos con situaciones de la vida real.

\begin{tabular}{cccccc}
\hline & Frecuencia & Porcentaje & Porcentaje válido & $\begin{array}{c}\text { Porcentaje } \\
\text { acumulado }\end{array}$ \\
\hline \multirow{4}{*}{ Válidos } & Muy de acuerdo & 43 & 21,0 & 21,0 & 21,0 \\
& De acuerdo & 119 & 58,0 & 58,0 & 79,0 \\
& Indeciso & 34 & 16,6 & 16,6 & 95,6 \\
& En desacuerdo & 6 & 2,9 & 2,9 & 98,5 \\
& Muy en desacuerdo & 3 & 1,5 & 1,5 & 100,0 \\
& Total & 205 & 100,0 & 100,0 & \\
\hline
\end{tabular}

Fuente: Elaboración propia.

Al observar el porcentaje acumulado de la tabla 2 se puede notar claramente que solo el $21,0 \%$ indican estar muy de acuerdo que el ABP se centra en aspectos problemáticos relacionados con la sociedad, mientras que el 95,6\% están indecisos con esta situación y muy cercano se encuentra el porcentaje del $98,5 \%$ que manifiestan en desacuerdo, porque consideran que las problemáticas a tratar no deben ser situaciones de la vida real, sino trabajar en proyectos antes establecidos. Tan solo el 79,0\% están de acuerdo con trabajar con este tipo de proyectos.

\section{Tabla 3.}

Cree que el ABP contribuye al trabajo interdisciplinario dentro de su institución educativa al que presta sus servicios. 
CIENCIAMATRIA

Revista Interdisciplinaria de Humanidades, Educación, Ciencia y Tecnología

Año VI. Vol. VI. N³. Edición Especial III. 2020

Hecho el depósito de ley: pp201602FA4721

ISSN-L: 2542-3029; ISSN: 2610-802X

Universidad Nacional Experimental Francisco de Miranda (UNEFM). Santa Ana de Coro. Venezuela

Blanca Nieves Cárdenas-Contreras; Darwin Gabriel García-Herrera; María Isabel Álvarez-Lozano; Juan Carlos Erazo-Álvarez

\begin{tabular}{ccccc}
\hline & Frecuencia & Porcentaje & Porcentaje válido & $\begin{array}{c}\text { Porcentaje } \\
\text { acumulado }\end{array}$ \\
\hline Muy de acuerdo & 23 & 11,2 & 11,2 & 11,7 \\
De acuerdo & 104 & 50,7 & 50,7 & 62,4 \\
Indeciso & 63 & 30,7 & 30,7 & 93,2 \\
En desacuerdo & 11 & 5,4 & 5,4 & 98,5 \\
Muy en desacuerdo & 3 & 1,5 & 1,5 & 100,0 \\
\hline Total & 205 & 100,0 & 100,0 & \\
\hline
\end{tabular}

Fuente: Elaboración propia.

Al considerar el ABP, dentro de la interdisciplinaridad, los estudiantes están muy de acuerdo con un porcentaje acumulado de $11,7 \%$ y de acuerdo el $62,4 \%$ lo que indica que esta metodología puede aplicarse dentro de otras asignaturas del pensum académico, en tanto que el indeciso se encuentra con un $93,2 \%$ y en desacuerdo el $98,5 \%$, lo que da a entender que el estudiante desconoce que los temas y actividades, se encuentran relacionadas con las demás asignaturas. Es labor entonces del docente reiterar continuamente que todas las asignaturas son interdisciplinarias y que no pueden trabajarse por separadas. 
CIENCIAMATRIA

Revista Interdisciplinaria de Humanidades, Educación, Ciencia y Tecnología

Año VI. Vol. VI. N³. Edición Especial III. 2020

Hecho el depósito de ley: pp201602FA4721

ISSN-L: 2542-3029; ISSN: 2610-802X

Universidad Nacional Experimental Francisco de Miranda (UNEFM). Santa Ana de Coro. Venezuela

Blanca Nieves Cárdenas-Contreras; Darwin Gabriel García-Herrera; María Isabel Álvarez-Lozano; Juan Carlos Erazo-Álvarez

\section{Tabla 4.}

Relación entre en las clases de Ciencias sociales, los docentes aplican metodologías activas al momento de enseñar y el aprendizaje Basado en proyectos es una estrategia metodológica que fomenta la elaboración de proyectos con situaciones de la vida real.

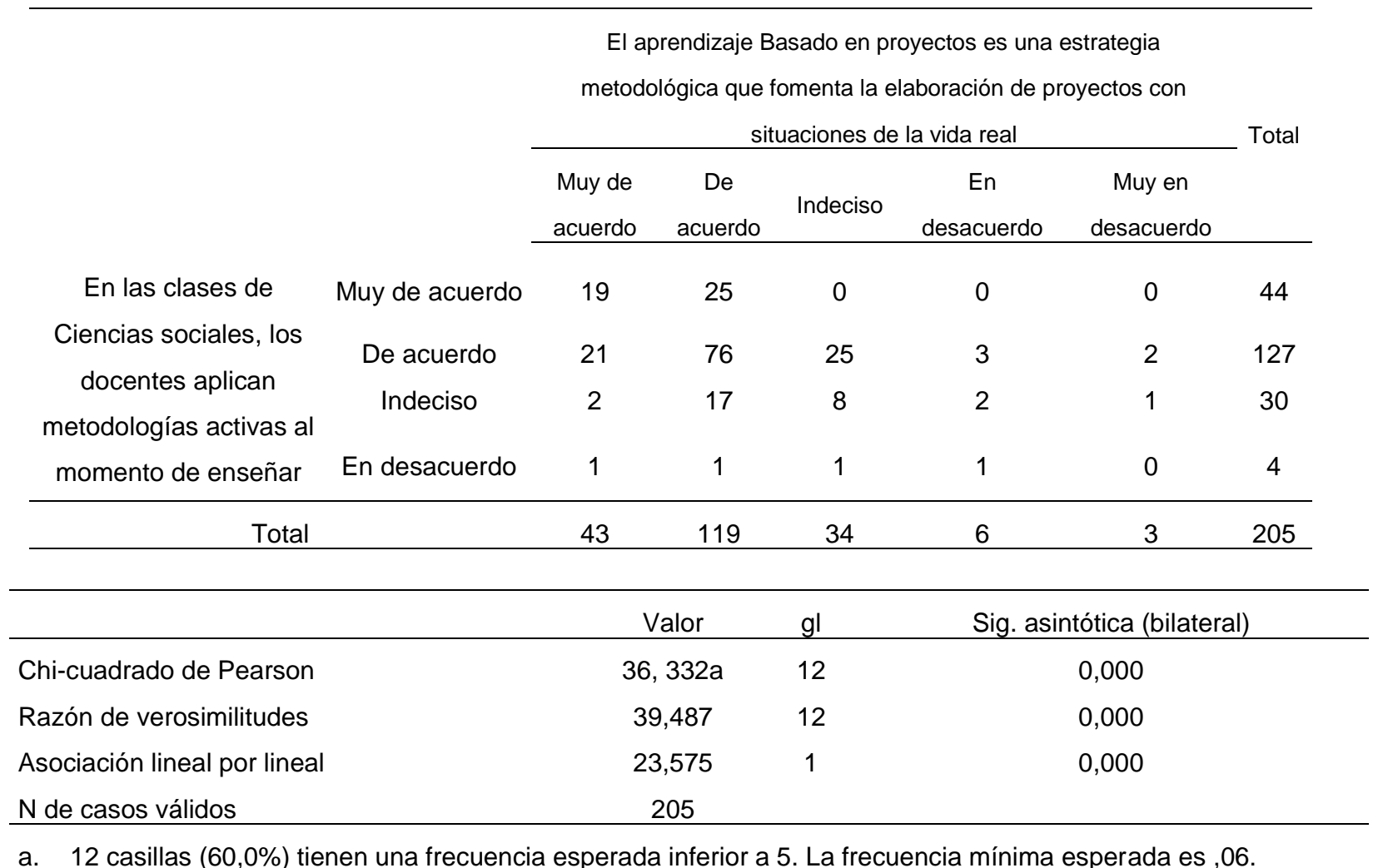

Fuente: Elaboración propia. 
Universidad Nacional Experimental Francisco de Miranda (UNEFM). Santa Ana de Coro. Venezuela

Blanca Nieves Cárdenas-Contreras; Darwin Gabriel García-Herrera; María Isabel Álvarez-Lozano; Juan Carlos Erazo-Álvarez

Luego de haber realizado el respectivo análisis de la tabla 4 acerca del Aprendizaje Basado en Proyectos es una estrategia metodológica que fomenta la elaboración de proyectos con situaciones de la vida real, el valor de Chi-cuadrado de Pearson es menor al valor crítico, por lo que, el objetivo es afirmativo, lo que equivale a $H 1$. Determinando la relación que tiene con la interrogante ¿En las clases de Ciencias Sociales los docentes aplican metodologías activas al momento de enseñar? las dos variables son compatibles, por cuanto la relación es directa, esto permite hacer uso de metodologías activas como el Aprendizaje Basado en Proyectos, para mejorar el rendimiento académico de los estudiantes en la disciplina de Ciencias Sociales.

Por lo tanto, según la escala de acuerdo, se puede observar que el porcentaje de respuestas es alto, en comparación con el de muy de acuerdo e indeciso donde los valores son paralelos o cercanos; no así, en la escala de desacuerdo en donde se puede apreciar que los estudiantes consideran que no es factible aplicar esta metodología dentro de Ciencias Sociales.

\section{Tabla 5.}

En las clases de Ciencias Sociales, la aplicación de metodologías activas ayuda a seguir manteniendo las técnicas tradicionales.

\begin{tabular}{cccccc}
\hline & & Frecuencia & Porcentaje & Porcentaje válido & $\begin{array}{c}\text { Porcentaje } \\
\text { acumulado }\end{array}$ \\
\hline \multirow{3}{*}{ Válidos } & Muy de acuerdo & 2 & 13,3 & 13,3 & 13,3 \\
& De acuerdo & 5 & 33,3 & 33,3 & 46,7 \\
& Indeciso & 1 & 6,7 & 6,7 & 53,3 \\
& En desacuerdo & 4 & 26,7 & 26,7 & 80,0 \\
& Muy en desacuerdo & 3 & 20,0 & 20,0 & 100,0 \\
& Total & 15 & 100,0 & 100,0 & \\
\hline
\end{tabular}

Fuente: Elaboración propia. 
Universidad Nacional Experimental Francisco de Miranda (UNEFM). Santa Ana de Coro. Venezuela

Blanca Nieves Cárdenas-Contreras; Darwin Gabriel García-Herrera; María Isabel Álvarez-Lozano; Juan Carlos Erazo-Álvarez

Al revisar el porcentaje acumulado de la tabla 5 de los docentes se puede apreciar que $13,3 \%$ se encuentran muy de acuerdo que, en las clases de Ciencias Sociales, la aplicación de metodologías activas ayuda a seguir manteniendo las técnicas tradicionales, mientras que el 46,7 están de acuerdo, al igual que los indecisos con un porcentaje de $53,3 \%$,por lo que se puede notar que muchos docentes piensan que estas metodologías activas siguen manteniendo un tradicionalismo en las aulas de clases, pero $80,0 \%$ de los encuestados indican que están totalmente en desacuerdo ya que las metodologías activas como es el Aprendizaje Basado en Proyectos ayuda a dejar atrás aquel tradicionalismo dentro de la educación, fomentando así el modernismo como lo proponen las nuevas escuelas pedagógicas basadas en el constructivismo de Dewey.

\section{Tabla 6.}

El Aprendizaje Basado en Proyectos estimula el aprendizaje colaborativo y cooperativo en el proceso enseñanza aprendizaje de los estudiantes en Ciencias Sociales.

\begin{tabular}{cccccc}
\hline & & Frecuencia & Porcentaje & Porcentaje válido & $\begin{array}{c}\text { Porcentaje } \\
\text { acumulado }\end{array}$ \\
\hline \multirow{3}{*}{ Válidos } & Muy de acuerdo & 9 & 60,0 & 60,0 & 60,0 \\
& De acuerdo & 6 & 40,0 & 40,0 & 100,0 \\
& Total & 15 & 100,0 & 100,0 & \\
\hline
\end{tabular}

Fuente: Elaboración propia.

Al pensar que el ABP estimula el aprendizaje colaborativo y cooperativo en el proceso enseñanza de los estudiantes en Ciencias Sociales ,el 60\% de los docentes indican que están muy de acuerdo, puesto que esta metodología si propicia la colaboración y cooperación entre los estudiantes al realizar sus proyectos, en cambio el $40 \%$ manifiestan que están de acuerdo, por lo que se deduce que este tipo de aprendizaje, contribuye al trabajo en equipo.

Como se demuestra en estudios realizados en Colombia, donde se pudo comprobar que, mediante la aplicación de un proyecto, basado en la metodología Aprendizaje Basado en 
Universidad Nacional Experimental Francisco de Miranda (UNEFM). Santa Ana de Coro. Venezuela

Blanca Nieves Cárdenas-Contreras; Darwin Gabriel García-Herrera; María Isabel Álvarez-Lozano; Juan Carlos Erazo-Álvarez

Proyectos, existió una ruptura con la tradicional enseñanza, puesto que el estudiante participa activamente en el desarrollo del mismo y el docente paso a ser un guía y facilitador, demandando cambios en toda la comunidad educativa. (Ciro, 2012)

Incluso (Martí, Heydrich, Rojas, \& Hernández, 2010) indica que en México también se trabajó con esta estrategia metodológica en las asignaturas de Ecología Microbiana, permitiendo a los estudiantes adquirir destrezas, habilidades a fin de integrar la teoría con la práctica, donde se dejó de lado aquella enseñanza mecánica, para orientarse en un trabajo más retador, utilizando el trabajo cooperativo de los participantes, teniendo muy buenos resultados.

Es importante recalcar que el uso del Aprendizaje Basado en Proyectos y las demás metodologías activas debe estar acompañado de las herramientas digitales que ofrece el mundo tecnológico en la actualidad ya que la prioridad de los docentes debe ser la implementación de las TIC y saber cómo utilizar pedagógicamente en sus distintas asignaturas pertenecientes a Ciencias Sociales, con el fin de obtener información muy relevante, que permitan mejorar la calidad educativa en la institución de estudio como es la Unidad Educativa Luis Rogerio González de la ciudad de Azogues. (Sierra, Bueno, \& Monroy, 2016)

\section{PROPUESTA}

Luego de haber revisado el tema de investigación se ha analizado algunas herramientas digitales que ayudarán a mejorar la utilización, de la estrategia metodológica, Aprendizaje Basado en Proyectos, para la enseñanza de Ciencias Sociales en el bachillerato, por lo que se sugiere algunas opciones que el docente podrá utilizar de manera adecuada, venciendo el tabú a los recursos digitales y adecuándose a las necesidades de los estudiantes. Entre las herramientas digitales que se han considerado indispensables en la enseñanza de esta disciplina en bachillerato son las siguientes: 
Universidad Nacional Experimental Francisco de Miranda (UNEFM). Santa Ana de Coro. Venezuela

Blanca Nieves Cárdenas-Contreras; Darwin Gabriel García-Herrera; María Isabel Álvarez-Lozano; Juan Carlos Erazo-Álvarez

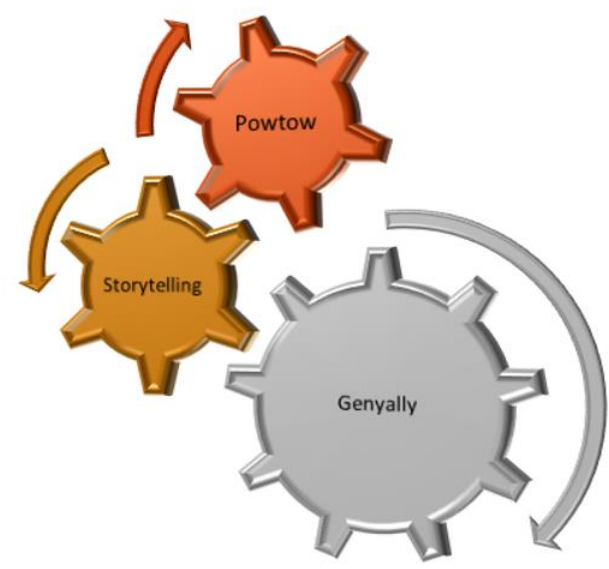

Figura 1. Propuesta recursos didácticos para la endeñanza de Ciencias Sociales. Fuente: Elaboración propia.

Powtow: Es un recurso didáctico educativo online, que permite crear todo tipo de presentaciones animadas en video, con personajes, caricaturas, música, textos y objetos, etc. Es magnifico para despertar el interés en los alumnos, al crear sus propias historias, siendo parte de los acontecimientos narrados, ya que están muy relacionados con la era tecnológica, que ofrece múltiples ventajas como: Presentaciones rápidas, permitiendo al estudiante usar todas las preeminencias que ofrece la misma, con el fin de que él construya su propia historia, mediante un proyecto de clases especialmente en la asignatura de Ciencias Sociales, ya que el estudiante aprende participando y siendo parte de los acontecimientos, a través de esta herramienta digital.

Incluso brinda efectos de textos, imágenes con transiciones, por cuanto inserta personajes, objetos y formas que ya vienen predefinidos, para no tener complicaciones, al momento de utilizarlo. Además permite, realizar presentaciones didácticas, dinámicas y de facil comprención, con un método muy sencillo,donde se puede utilizar la voz y música para darle más realismo al mensaje ¡Dile adiós a la diapositiva aburrida y saturada de texto! Con Powtoon aprenderás a comunicar de forma divertida. Algo muy sorprendente es que una vez finalizado el proyecto, tinene la posibilidad de compartir la 
Universidad Nacional Experimental Francisco de Miranda (UNEFM). Santa Ana de Coro. Venezuela

Blanca Nieves Cárdenas-Contreras; Darwin Gabriel García-Herrera; María Isabel Álvarez-Lozano; Juan Carlos Erazo-Álvarez

creación en las redes sociales para que más gente se entere de su trabajo, redes como Facebook y You tube.

Otra de las ventajas del uso de esta harramienta digital, es que ayuda a las personas con discapacidades, en el caso de los no videntes y los que tienen un coeficinte intelectual bajo, puesto que ellos ,manejan a través del braille la tegnología con estos recursos didácticos, mejorando su aprendizaje ya que ellos pueden grabar su voz y elaborar su presentación con el tema que ellos consideren conveniente dentro de la disciplina de Ciencias Sociales, de igual manera con aquellos estudiantes que tienen problemas de aprendizaje, la utilización de este recurso les permite desarrollar su creatividad con las caricaturas que utliza en la contrucción de la historia,y sobre todo porque el es parte activa de la narración y son cosas que él nunca se va a olvidadar ya que fue el constructor de su propio aprendizaje.

Storytelling: Es una herramienta digital sugerida dentro del Aprendizaje Basado en Proyectos, para motivar la enseñanza de Ciencias Sociales en bachillerato a los estudiantes. Misma que sirve para contar historias utilizando la tecnología.

En las aulas escolares la digitalización y el aprendizaje ha dado una gran revolución a la hora de contar historias, donde la narración tradicional adquiere una nueva dimensión con el incremento de la tecnologia para transmitir conocimientos y valores que despierten el interés y la motivación de los alumnos, es una poderosa arma que puede utilizar el docente para que sus relatos sean inventados o reales.

Puesto que, ellos ya no contaran sus relatos de manera oral sino a través de las utilizaciones del storytelling, con el cual el estudiante quedará fascinado al contar su historia, de ahí la importancia del uso de esta herramienta en las clases sobre todo teóricas como lo es la historia. Mismas que proporciona a los alumnos motivación, diversión, curiosidad y actitudes favorables para el aprendizaje, ayuda a establecer relaciones entre los contenidos, organizando la información. 
Universidad Nacional Experimental Francisco de Miranda (UNEFM). Santa Ana de Coro. Venezuela

Blanca Nieves Cárdenas-Contreras; Darwin Gabriel García-Herrera; María Isabel Álvarez-Lozano; Juan Carlos Erazo-Álvarez

Ayuda a fomentar la creatividad, el pensamiento crítico, desarrolla habilidades, permite mejorar la atención y el nivel de concentración, crea un vínculo entre el docente y los estudiantes y facilita una comunicación fluida en donde genera un clima participativo de trabajo. Recurso necesario en el Aprendizaje Basado en Proyectos, mismo que facilita la construcción de proyectos, con el uso de la tecnología para sus auditorios, dejando atrás las presentaciones expositivas.

Genially: Hoy en día es fundamental dentro de educación aplicar nuevas estrategias, como el ABP vinculadas con la era digital, donde permita captar la atención de los estudiantes, utilizando tecnologías que les diviertan y sorprendan, siendo una de ellas Genially, que se destaca por la capacidad de crear contenidos con un alto grado de atractivo visual, generando una experiencia enriquecedora de aprendizaje, en donde los estudiantes, tendrán la oportunidad de presentar sus proyectos realizados dentro de Ciencias Sociales, de manera agradable.

Una de las ventajas que ofrece es, que se puede trabajar online y todas las creaciones se guardan automáticamente en la nube, facilitando realizar nuestra labor, desde cualquier lugar, fomenta dentro del aula un trabajo colaborativo y cooperativo, ya que facilita a varios usuarios editar un mismo recurso, resultando muy útil a la hora de hacer trabajos grupales, ya que ofrece una serie de plantillas para elaborar de forma rápida y sencilla contenidos atractivos y vistosos.

\section{CONCLUSIONES}

A partir del estudio realizado sobre la influencia del Aprendizaje Basado en Proyectos, como estrategia metodológica para la enseñanza de Ciencias Sociales en los estudiantes de bachillerato, se deduce que esta, ayuda a fomentar un aprendizaje dinámico, creativo, reflexivo y autosuficiente, en donde el estudiante busca alternativas para solucionar sus problemas, dejando atrás las clases tradicionales donde él receptaba solo conocimientos. 
Universidad Nacional Experimental Francisco de Miranda (UNEFM). Santa Ana de Coro. Venezuela

Blanca Nieves Cárdenas-Contreras; Darwin Gabriel García-Herrera; María Isabel Álvarez-Lozano; Juan Carlos Erazo-Álvarez

Según análisis emitido en las pruebas ser bachiller los niveles de aprendizaje en Ciencias Sociales es bajo en los jóvenes de bachillerato, razón por la cual se investigó metodológias innovadoras accesibles como el ABP, acompañado de recursos digitales, que mejorarán el hábito por aprender temas históricos y despertarán el interés dentro de esta disciplina.

Con los resultados analizados en la presente investigación se pudo establecer, como esta estrategia metodológica junto a recursos didácticos afianzados al campo tecnológico como: powtoow, storytelling y genially ayudan en la enseñanza de esta disciplina, de manera presencial y virtual, incentivando a los estudiantes al gusto por estas asignaturas, por ello se propone que trabaje toda la comunidad educativa con proyectos innovadores permitiendo de esa manera alcanzar los objetivos deseados en torno a la problemática identificada dentro del salón de clases.

Finalmente se comprobó que existen métodos muy dinámicos y creativos, que ayudan al discente a ser el protagonista de su aprendizaje, en donde el rol docente se convierte en facilitador del proceso, por ello nuestra recomendación a los docentes implementar metodológias y recursos tecnológicos, para hacer de las clases de Ciencias Sociales más productivas.

\section{REFERENCIAS CONSULTADAS}

Ardeleanu, R. (2019). Traditional and Modern Teaching Methods in Matematicas [Métodos de enseñanza tradicionales y modernos en Matematicas]. Scholarly Journals, 23(2), 134-135. Obtenido de https://n9.cl/4di4d

Arteaga , E., Arteaga, L., \& Martínez, J. (2016). La enseñanza de las ciencias en el nuevo milenio. Retos y sugerencias [Science education in the new millennium. Challenges and suggestions]. Universidad y Sociedad, 8(1), 170-171. Obtenido de https://n9.cl/7mku

Ardeleanu, R. (2019). Traditional and Modern Teaching Methods in Matematicas [Métodos de enseñanza tradicionales y modernos en Matematicas]. Scholarly Journals, 23(2), 134-135. Obtenido de https://n9.cl/4di4d 
Universidad Nacional Experimental Francisco de Miranda (UNEFM). Santa Ana de Coro. Venezuela

Blanca Nieves Cárdenas-Contreras; Darwin Gabriel García-Herrera; María Isabel Álvarez-Lozano; Juan Carlos Erazo-Álvarez

Ciro, C. (2012). Aprendizaje Basado en Proyectos (A.B.Pr) Como estrategia de Enseñanza y Aprendizaje en la Educación Básica y Media. Colombia: Universidad Nacional de Colombia. Obtenido de https://n9.cl/z86nt

Cunill, F. (2017). El Aprendizaje Basado en Proyectos y el Aprendizaje por descubrimiento Guiado como estrategias didacticas en Biologia yb Geologia de 40 de ESO [Project-Based Learning and Guided Discovery Learning as didactic strategies in Biology and Geology of 4th year]. Palma Mallorca. Obtenido de https://n9.cl/fy $75 t$

García-Varcálcel, A., \& Basilotta, V. (2017). Aprendizaje Basado en Proyectos (ABP): evaluación desde la perspectiva de alumnos de Educación Primaria [ProjectBased Learning (PBL): evaluation from the perspective of Primary Education students]. Revista de Investigacion Educativa, 1(35), 114-115. doi:http://dx.doi.org/10.6018/rie.35.1.246811

Gómez, C., \& Rodríguez, A. (2014). Aprender a enseñar ciencias sociales con métodos de indagación. Los estudios de caso en la formación del profesorado. redu, 12(2). doi:DOI: $\underline{10.4995 / \text { redu.2014.5651 }}$

Gómez, C., Ortuño, J., \& Millares, P. (2018). Ensenar Ciencias Sociales con metodos activos de aprendizaje [Teach Social Sciences with active learning methods]. Espana. Obtenido de https://n9.cl/s431

Habók, A., \& Nagy, J. (2016). In-service teachers' perceptions [Percepciones de los profesores en servicio]. SpringerPlus, 3-4. doi:DOI 10.1186/s40064-016-1725-4

León, O., Martinez, F., \& Santos, M. (2018). Análisis de la investigación sobre Aprendizaje basado en Proyectos en Educación Física [Research Analysis on Project-Based Learning in Physical Education]. Revista electroníca interuniversitaria de formación del profesorado., 28-29. doi: http://dx.doi.org/10.6018/reifop.21.2.323241

Martí, J., Heydrich, M., Rojas, M., \& Hernández, A. (2010). Aprendizaje basado en proyectos: una experiencia de innovación docente. Universidad EAFIT, 46(158), 20. Obtenido de https://www.redalyc.org/pdf/215/21520993002.pdf

Martinez, D. (2018). Ensenanza Trdicional en el siglo XXI [Trditional Teaching in the 21st century]. Neuronum, 4(1), 3-4. Obtenido de https://n9.cl/37kw 
Universidad Nacional Experimental Francisco de Miranda (UNEFM). Santa Ana de Coro. Venezuela

Blanca Nieves Cárdenas-Contreras; Darwin Gabriel García-Herrera; María Isabel Álvarez-Lozano; Juan Carlos Erazo-Álvarez

Medina, M., \& Tapia, M. (2017). El Aprendizaje Basado en Proyectos una oportunidad para trabajar [Project Based Learning an opportunity to work]. OLIMPIA, 14(46), 236-237. Obtenido de https://n9.cl/mfg7

Millares, P., Gómez, C., Arias, V., \& Fontal, O. (2019). Recursos digitales y metodología didáctica en la formación inicial de docentes de Historia [Digital resources and didactic methodology in the initial training of History teachers]. Comunicar, 27(61), 46-47. doi:https://doi.org/10.3916/C61-2019-04

Ministerio de Educación del Ecuador. (2019). Ministerio de Educación del Ecuador [Ministry of Education of Ecuador]. Obtenido de https://n9.cl/6ag3

Molina, M. (2019). El Aprendizaje Basado en Proyectos (abp) en la formación metodológica [Project-Based Learning (PBL) in methodological training]. EVSAL, 37(1), 124-125. doi:https://doi.org/10.14201/et2019371123137

Murillo, F. J., \& Perines, H. (2017). Cómo los docentes no universitarios perciben la investigación educativa [How non-university teachers perceive educational research]. Complutense de Educación, 28(1), 82-83. doi:http://dx.doi.org/10.5209/rev RCED.2017.v28.n1.48800

Neiva, A. A., Bandeira, M., Alencar, D., \& Magalhães, L. (2020). O uso de metodologia ativa no campo das Ciências Sociais em Saúde: relato de experiência de produção audiovisual por estudantes[Or use of active methodology in the field of Social Sciences in Health: experience report of audiovisual production by students. Interface, 24, 3. doi:https://doi.org/10.1590/interface.190231

Orozco, J. (2016). Estrategias Didácticas y aprendizaje de las Ciencias Sociales [Didactic Strategies and Learning of Social Sciences]. FAREM, 5(17), 76. Obtenido de https://n9.cl/3bc0x

Romero, A., Forero, A., \& Rodríguez, A. (2018). Análisis comparación del aprendizaje basado en proyectos de forma tradicional y con mediación de las TIC [Comparison analysis of project-based learning in a traditional way and with the mediation of ICT]. Espacios, 39(52). Obtenido de https://n9.cl/pxe3

Romero, A., Forero, A., \& Rodríguez, A.-A. (2018). Análisis comparación del aprendizaje basado en proyectos de forma tradicional y con mediación de las TIC [Comparison analysis of project-based learning in a traditional way and with the mediation of ICT]. Espacios, 39(52). Obtenido de https://n9.cl/it7q 
Universidad Nacional Experimental Francisco de Miranda (UNEFM). Santa Ana de Coro. Venezuela

Blanca Nieves Cárdenas-Contreras; Darwin Gabriel García-Herrera; María Isabel Álvarez-Lozano; Juan Carlos Erazo-Álvarez

Ruiz, M., \& Gómez, I. (2017). Herramientas innovadoras para futuros docentes de enseñanza Secundaria [Innovative tools for future secondary school teachers]. España: Universidad Alicante. Obtenido de https://n9.cl/4nv66

Sánchez, A. (2018). La metodología ABP y su aplicación a las Ciencias Sociales. Obtenido de https://n9.cl/pg4j2

Sierra, J., Bueno, I., \& Monroy, S. (2016). Análisis del uso de las tecnologías TIC por parte de los docentes de las Instituciones educativas de la ciudad de Riohacha [Analysis of the use of ICT technologies by teachers of educational institutions in the city of Riohacha]. OMNIA, 22(2). Obtenido de https://n9.cl/1ahj0

Valdivia, S., \& Cobo, G. (2017). Aprendizaje Basado en Proyectos [Project Based Learning]. Perú: Pontificia Universidad Católica del Perú. Obtenido de https://n9.cl/sobi 